\title{
The pancreas in health and in diabetes
}

\section{Sally M. Marshall ${ }^{1}$}

Published online: 31 August 2020

(C) Springer-Verlag GmbH Germany, part of Springer Nature 2020

The first recognition of the pancreas as an organ is accredited to Herophilus of Chalcedon, often called the father of anatomy, more than 2000 years ago [1]. The functions of the organ remained a mystery until Claude Bernard's work on pancreatic juice and digestion in the late 19th century [2]. Interest in and work on the pancreas then rapidly accelerated. Everyone in the diabetes field is aware of the description of the islets of Langerhans within the pancreas by the medical student Paul Langerhans in 1869 [3], followed by the demonstration that total pancreatectomy precipitated diabetes mellitus by von Mering and Minkowski in 1889 [4]. Although others had come close to isolating the pancreatic secretion necessary to prevent diabetes mellitus, it was Frederick Banting and Charles Best, ably aided by biochemist James Collip, in the laboratories of J. J. R. Macleod in Toronto, Canada, who eventually succeeded [5]. Details of the story of the discovery are elegantly described by Michael Bliss [6].

The complexity of the pancreas was appreciated early. Alpha and beta cells in the islets of Langerhans were distinguished by conventional histological techniques in 1907 [7], but it wasn't until 1959 that localisation of insulin to the beta cell was confirmed [8]. Likewise, although glucagon was discovered in 1923, it was localised to the alpha cells in 1962 [9]. Since then, a whole raft of new scientific techniques have allowed detailed examination of the anatomy and function of the pancreas, the discovery of other hormones within the islets of Langerhans, and detailed study of the complex interactions between different cells within the pancreas, and between the pancreas and other organs in the body. This special issue offers insight into many of these recent advances, challenging many long-held concepts and providing clues to future important areas for work.

Sally M. Marshall

sally.marshall@newcastle.ac.uk

1 Diabetes Research Group, Translational and Clinical Research Institute, Faculty of Clinical Medical Sciences, Newcastle University, 4th Floor William Leech Building, Framlington Place, Newcastle upon Tyne NE2 4HH, UK
Atkinson et al [10] launch the series with a review of the current evidence on human pancreatic anatomy as it pertains to endocrine and exocrine function, and the changes that occur in individuals with type 1 or type 2 diabetes.

Jennings and colleagues [11] go on to discuss how alterations in important steps in the development of the mammalian pancreas may contribute to the abnormalities seen in diabetes. For example, mutations in specific transcription factors that help to shape the mammalian pancreas have been shown to result in pancreas agenesis, absence of islet formation or absence of beta cells in mice, with similar phenotypes being recapitulated in human model systems or individuals with neonatal diabetes.

Post-pancreas development, abnormal insulin production by the pancreas underlies the pathogenesis of diabetes. Yet many aspects of insulin biosynthesis in pancreatic islet beta cells remain elusive. On the other hand, increasing evidence demonstrates that deficits in insulin production contribute to the pathogenesis of both type 1 and type 2 diabetes, as well as monogenetic forms of this disease. In their review, Vasiljević and coauthors [12] outline the most recent findings about the post-transcriptional mechanisms regulating insulin production and how their deficits can cause diabetes.

The sole source of circulating insulin is the pancreatic beta cell. In their review, Rutter et al [13] discuss the specialisations that allow this cell to perform its unique role. Glucose metabolism is key to the activation of beta cell secretion thanks to numerous modifications to the metabolic pathways seen in most other cells. These include high expression of glucose transporters and glucokinase, and selective disallowance of other genes, such as $L D H A$ and SLC16A1. The authors also explain how abnormal oxidative metabolism by mitochondria is often observed in type 2 diabetes, suggesting a critical role for mitochondrial $\mathrm{Ca}^{2+}$, while deletion of certain genes, such as $\mathrm{Mcu}$ (encoding the mitochondrial $\mathrm{Ca}^{2+}$ uniporter), inhibits insulin secretion.

Autoimmune beta cell destruction results in type 1 diabetes. But why are beta cells vulnerable autoimmune targets? Mallone and Eizirik [14] aim to answer this question by discussing how autoimmune vulnerability may be linked to some biological features of the beta cell, including the cellular 
stress associated with the synthesis of insulin and other granule proteins, the rich vascularisation of islets and the secretion of beta cell products directly into the bloodstream. According to the authors, appreciation that beta cells are integral drivers in the pathogenesis of type 1 diabetes has key implications for the discovery of circulating signatures of beta cell stress that can complement autoimmune biomarkers, and for the development of novel therapeutic strategies that can combine immunomodulatory and beta cell-protective agents according to disease endotypes.

Beta cells also play a role in the development of type 2 diabetes, and it is well accepted that beta cell dysfunction is required for hyperglycaemia to occur. The prevailing view is that, in the presence of insulin resistance, beta cell dysfunction that occurs early in the course of the disease process is the critical abnormality in type 2 diabetes. Recently, an alternative model has been proposed in which primary beta cell overstimulation results in insulin hypersecretion that then leads to the development of obesity and insulin resistance, and ultimately to beta cell exhaustion. In their review, Esser et al [15] discuss data from preclinical and clinical studies in the context of these models and outline which model is more likely to explain the pathogenesis of hyperglycaemia in type 2 diabetes.

Since age is one of the main risk factors for developing type 2 diabetes, it is also important to understand the effects of ageing on beta cell function. Cristina Aguayo-Mazzucato [16] summarises the functional changes that occur in ageing beta cells. Insulin secretion from beta cells decreases as humans age, with recent publications pinpointing senescence, a cellular stress response, as one of the mechanisms behind this functional loss. However, the author outlines how senolysis (the specific removal of senescent cells) is a promising approach to reverse some of the changes induced by age, resulting in beta cell functional recovery and improved insulin secretion.

Diabetes may also occur secondary to exocrine pancreatic disease, leading to pancreatogenic (or type 3c) diabetes. As discussed by Rickels et al [17], diabetes is a common consequence of a wide variety of exocrine pancreas diseases, ranging from monogenetic disorders of the exocrine pancreas, such as cystic fibrosis and MODY type 8 , to chronic pancreatitis and pancreatic ductal adenocarcinoma. The authors explain that, although these conditions are all classified as forms of pancreatogenic diabetes, there are shared and unique aspects of the molecular physiology of islet failure and diabetes across the specific forms.

In fact, heterogeneity does not just exist within pancreatogenic diabetes, but is also evident within and between other diabetes types, as outlined by Redondo et al [18]. The authors explain that endotypes have been defined within type 1 diabetes, with implications for disease prediction, prevention, diagnosis and treatment, while in type 2 diabetes subgroups relevant to prognosis and treatment have been identified. The blurring of the classic distinctions between diabetes types has driven efforts to define additional entities as distinct; Redondo and colleagues propose a paradigm that combines the 'palette model' and the 'threshold hypothesis' to help explain the heterogeneity within and between diabetes types, concluding that this new approach may facilitate personalised medicine in diabetes.

For example, in patients with type 1 diabetes complicated by life-threatening severe hypoglycaemia or renal failure requiring kidney transplantation, a whole-organ pancreas or islet transplant may be optimal to restore normoglycaemia, eliminate severe hypoglycaemia and restore insulin independence. In their review, Bellin and Dunn [19] discuss the variety of transplant strategies available for individuals with type 1 diabetes, but note that transplantation is currently restricted to a small subset of the patient population with type 1 diabetes because human pancreas or islet transplantations rely on the availability of pancreases from deceased donors. However, the authors discuss how porcine islets, which can successfully reverse diabetes in non-human primate models, offer a potential unlimited source of islets to treat patients with type 1 diabetes in the future.

Looking more closely at the islet itself, Mark Huising [20] discusses the cellular components of this special structure that are important to its role. To this day, many people in the diabetes field equate pancreatic islets with beta cells. Indeed, they are the most numerous cell type in the islet and their loss or dysfunction causes diabetes. However, Huising highlights how healthy islets precisely control glucose levels over decades via carefully coordinated local interactions between beta, alpha and delta cells. While, under many normal physiological conditions, glucose remains the sine qua non of insulin secretion, a rich chorus of local interactions amplifies, modulates and curtails the stimulatory effects of nutrients on beta cells.

Moede et al [21] go on to focus specifically on the importance of islet alpha cell-beta cell communication for glucose homeostasis. The authors describe some of the early observations of the positive effect of alpha cells and glucagon on glucose-stimulated insulin secretion from beta cells, as well as more recent studies investigating the in vivo role of paracrine glucagon and glucagon-like peptide 1 signalling. Their review also discusses the role of the alpha cell-derived neurotransmitter acetylcholine for human beta cell function. Looking to the future, the authors discuss the use of the anterior chamber of the mouse eye as a transplantation site for non-invasive, longitudinal, in vivo monitoring of paracrine alpha cell-beta cell signalling in mouse and human islets in health and disease.

It must be noted that endocrine cells in pancreatic islets do not live in a vacuum. Islets are also populated by vascular- and immune-cell populations, immersed in a three dimensional network of extracellular matrix. In their review, Almaça et al 
[22] summarise how different cellular and acellular components of the islet microenvironment can have an impact on endocrine cell function. The authors discuss recent findings on the interactions between pericytes and beta cells, illustrating how the microenvironment and beta cells influence each other. They propose that disruption of the integrity of the islet niche contributes to beta cell failure and diabetes pathogenesis.

As well as intra-pancreatic interactions, the stability of blood glucose levels depends upon the integrated actions of multiple organs that precisely coordinate glucose production and glucose uptake. For example, increasing evidence suggests that the central nervous system, working in collaboration with the islet, plays an essential role in glucose homeostasis. Faber et al [23] summarise both the anatomy of autonomic nervous system input to the islet and the functional importance of autonomic nervous system outflow to the islet across a variety of physiological challenges to glucose homeostasis. In addition, the authors examine mechanisms by which the blood glucose level is detected by the brain and discuss the potential consequences of defective brain glucose sensing for people with either type 1 or type 2 diabetes.

Efforts to phenotype pancreatic islets have contributed tremendously to our present understanding of endocrine function and diabetes. Recent advances in single-cell profiling have re-ignited interest in understanding islet and beta cell heterogeneity. While heterogeneity in secretory function has been known for decades, the links between heterogenous gene expression, cellular activities and insulin secretion are not well studied. To conclude our special series, Arrojo e Drigo et al [24] discuss approaches to connect molecular profiles with functional properties underlying insulin secretion. The contribution of spatio-temporal aspects is also considered. For example, ageing may impact the relative abundance of different beta cell subpopulations or states. Despite recent advances in knowledge, the authors propose that understanding the impact of molecular phenotypes on islet function is likely to require a 'scale up' of efforts focused on the multi-modal profiling of human islet preparations; several groups are making important steps in this direction. They conclude that, to better understand the contribution of islet phenotypes to metabolic homeostasis and diabetes risk, much work remains to connect in vitro and in vivo studies of human phenotypes.

I hope you will agree that the reviews in this special edition demonstrate the rich evidence base that has developed with regard to the pancreas and its role in health and diabetes. However, they also clearly illustrate the areas where we have only scratched the surface and our knowledge is incomplete. As always, I very much hope that we are all stimulated to continue to develop techniques in all disciplines to allow further progress to be made.

\section{References}

1. Fitzgerald PJ (1980) Medical anecdotes concerning some diseases of the pancreas. In: Fitzgerald PJ, Morrison AB (eds) The pancreas. Williams and Wilkins, Baltimore, pp 1-29

2. Olmsted JMD (1953) Claude Bernard, 1813-1879. Diabetes 2:162164. https://doi.org/10.2337/diab.2.2.162

3. Langerhans P (1869) Beiträge zur mikroskopischen Anatomie der Bauchspeicheldrüse. Gustav Lange, Berlin (Dissertation) [article in German]

4. Von Mering J, Minkowski O (1890) Diabetes mellitus nach pankreas extirpation. Arch Exp Pathol Pharmacol 26:371-387 [article in German]

5. Banting FG, Best CH, Collip JB, Campbell WR, Fletcher AA (1922) Pancreatic extracts in the treatment of diabetes mellitus: preliminary report. Can Med Assoc J 12:141-146

6. Bliss M (1982) The discovery of insulin. University of Chicago Press, Chicago

7. Lane MA (1907) The cytological characters of the areas of Langerhans. Am J Anat 7:409-421. https://doi.org/10.1002/aja. 1000070304

8. Lacy PE (1959) Electron microscopic and fluorescent antibody studies on the islets of Langerhans. Exp Cell Res 7:296-308. https://doi.org/10.1016/0014-4827(59)90250-2

9. Baum J, Simmons BE, Unger RH, Madison LL (1962) Localization of glucagon in the alpha cells in the pancreatic islet by immunofluorescent technics. Diabetes 11:371-374

10. Atkinson MA, Campbell-Thompson M, Kusmartseva I, Kaestner KH (2020) Organisation of the human pancreas in health and in diabetes. Diabetologia. https://doi.org/10.1007/s00125-020-05203-7

11. Jennings RE, Scharfmann R, Staels W (2020) Transcription factors that shape the mammalian pancreas. Diabetologia. https://doi.org/ 10.1007/s00125-020-05161-0

12. Vasiljević J, Torkko JM, Knoch KP, Solimena M (2020) The making of insulin in health and disease. Diabetologia. https://doi. org/10.1007/s00125-020-05192-7

13. Rutter GA, Georgiadou E, Martinez-Sanchez A, Pullen TJ (2020) Metabolic and functional specialisations of the pancreatic beta cell: gene disallowance, mitochondrial metabolism and intercellular connectivity. Diabetologia. https://doi.org/10.1007/s00125-02005205-5

14. Mallone R, Eizirik DL (2020) Presumption of innocence for beta cells: why are they vulnerable autoimmune targets in type 1 diabetes? Diabetologia. https://doi.org/10.1007/s00125-020-05176-7

15. Esser N, Utzschneider KM, Kahn SE (2020) Early beta cell dysfunction vs insulin hypersecretion as the primary event in the pathogenesis of dysglycaemia. Diabetologia. https://doi.org/10. 1007/s00125-020-05245-x

16. Aguayo-Mazzucato C (2020) Functional changes in beta cells during ageing and senescence. Diabetologia. https://doi.org/10. 1007/s00125-020-05185-6

17. Rickels MR, Norris AW, Hull RL (2020) A tale of two pancreases: exocrine pathology and endocrine dysfunction. Diabetologia. https://doi.org/10.1007/s00125-020-05210-8

18. Redondo MJ, Hagopian WA, Oram R et al (2020) The clinical consequences of heterogeneity within and between different diabetes types. Diabetologia. https://doi.org/10.1007/s00125-02005211-7

19. Bellin MD, Dunn TB (2020) Transplant strategies for type 1 diabetes: whole pancreas, islet and porcine beta cell therapies. Diabetologia. https://doi.org/10.1007/s00125-020-05184-7

20. Huising MO (2020) Paracrine regulation of insulin secretion. Diabetologia. https://doi.org/10.1007/s00125-020-05213-5 
21. Moede T, Leibiger IB, Berggren PO (2020) Alpha cell regulation of beta cell function. Diabetologia. https://doi.org/10.1007/s00125020-05196-3

22. Almaça J, Caicedo A, Landsman L (2020) Beta cell dysfunction in diabetes: the islet microenvironment as an unusual suspect. Diabetologia. https://doi.org/10.1007/s00125-020-05186-5

23. Faber CL, Deem JD, Campos CA, Taborsky GJ Jr, Morton GJ (2020) CNS control of the endocrine pancreas. Diabetologia. https://doi.org/10.1007/s00125-020-05204-6
24. Arrojo e Drigo R, Roy B, MacDonald PE (2020) Molecular and functional profiling of human islets: from heterogeneity to human phenotypes. Diabetologia. https://doi.org/10.1007/s00125-02005159-8

Publisher's note Springer Nature remains neutral with regard to jurisdictional claims in published maps and institutional affiliations. 